\title{
BITWISTED BURNSIDE-FROBENIUS THEOREM AND DEHN CONJUGACY PROBLEM
}

\author{
ALEXANDER FEL'SHTYN \\ Instytut Matematyki, Uniwersytet Szczeciński, ul. Wielkopolska 15, 70-451 Szczecin, Poland \\ and Boise State University, 1910 University Drive, Boise, Idaho, 83725-1555, U.S.A. \\ E-mail: felshtyn@diamond.boisestate.edu
}

\begin{abstract}
It is proved for Abelian groups that the Reidemeister coincidence number of two endomorphisms $\phi$ and $\psi$ is equal to the number of coincidence points of $\widehat{\phi}$ and $\widehat{\psi}$ on the unitary dual, if the Reidemeister number is finite. An affirmative answer to the bitwisted Dehn conjugacy problem for almost polycyclic groups is obtained. Finally, we explain why the Reidemeister numbers are always infinite for injective endomorphisms of Baumslag-Solitar groups.
\end{abstract}

\section{Introduction}

Definition 1.1. Let $G$ be a countable discrete group and $\phi, \psi: G \rightarrow G$ two endomorphisms. Two elements $x, x^{\prime} \in G$ are said to be $(\phi, \psi)$-conjugate iff there exists $\gamma \in G$ with

$$
x^{\prime}=\psi(\gamma) x \phi(\gamma)^{-1}
$$

The number of $(\phi, \psi)$-conjugacy classes is called the Reidemeister coincidence number of endomorphisms $\phi$ and $\psi$, denoted by $R(\phi, \psi)$. If $\psi$ is the identity map then the $(\phi, i d)$ conjugacy classes are the $\phi$-conjugacy classes in the group $G$ and $R(\phi, i d)=R(\phi)$ is the Reidemeister number of $\phi$. If both $\phi$ and $\psi$ are the identity maps then the $(i d, i d)$ conjugacy classes are the usual conjugacy classes in the group $G$. We shall write $\{x\}_{\phi, \psi}$ for the $(\phi, \psi)$-conjugacy or bitwisted conjugacy class of the element $x \in G$.

If $G$ is a finite group, then the classical Burnside-Frobenius theorem (see e.g. [25]) says that the number of classes of irreducible representations is equal to the number of conjugacy classes of elements of $G$. Let $\widehat{G}$ be the unitary dual of $G$, i.e. the set of equivalence classes of unitary irreducible representations of $G$.

2000 Mathematics Subject Classification: 20C, 20E45, 22D10, 22D25, 37C25, 43A30, 46L, 47H10, $54 \mathrm{H} 25,55 \mathrm{M} 20$.

Key words and phrases: Reidemeister number, bitwisted conjugacy classes, bitwisted conjugacy separable group, Burnside-Frobenius theorem.

The paper is in final form and no version of it will be published elsewhere. 
If $\phi: G \rightarrow G$ is an automorphism, it induces a map $\widehat{\phi}: \widehat{G} \rightarrow \widehat{G}, \widehat{\phi}(\rho)=\rho \circ \phi$. Therefore, by the Burnside-Frobenius theorem, if $\phi$ and $\psi$ are the identity automorphisms of any finite group $G$, then we have $R(\phi, \psi)=\# \operatorname{Coin}(\widehat{\phi}, \widehat{\psi})$, where $\operatorname{Coin}(\widehat{\phi}, \widehat{\psi})$ is the set of coincidence points of $\widehat{\phi}$ and $\widehat{\psi}$.

This statement remains true for $\phi \neq \mathrm{Id}, \psi=I d$ and finite $G$ [10]. Indeed, consider an automorphism $\phi$ of a finite group $G$. Then $R(\phi)$ is equal to the dimension of the space of twisted invariant functions on this group. Hence, by the Peter-Weyl theorem (which asserts the existence of a two-side equivariant isomorphism $\left.C^{*}(G) \cong \bigoplus_{\rho \in \widehat{G}} \operatorname{End}\left(H_{\rho}\right)\right)$, $R(\phi)$ is identified with the sum of dimensions $d_{\rho}$ of twisted invariant elements of $\operatorname{End}\left(H_{\rho}\right)$, where $\rho$ runs over $\widehat{G}$, and the space of representation $\rho$ is denoted by $H_{\rho}$. By the Schur lemma, $d_{\rho}=1$, if $\rho$ is a fixed point of $\widehat{\phi}$, and is zero otherwise. Hence, $R(\phi)$ coincides with the number of fixed points of $\widehat{\phi}$.

The attempts to generalize the Burnside-Frobenius theorem to the case of non-identical endomorphisms and of non-finite group were inspired by the dynamical questions and were the subject of a series of papers [6], [10], [13], [14], [15].

REMARK 1.2. If $\phi: G \rightarrow G$ is an epimorphism, it induces a map $\widehat{\phi}: \widehat{G} \rightarrow \widehat{G}, \widehat{\phi}(\rho)=\rho \circ \phi$ (because a representation is irreducible if and only if the scalar operators in the space of representation are the only ones which commute with all operators of the representation). This is also true for a general endomorphism of an Abelian group $G$, but is not the case for a general endomorphism $\phi$ of any group.

The paper consists of five sections. To make the presentation more detailed and transparent we start in Section 2 from a new approach for compact groups and two automorphisms. We develop this approach in Sections 3 and 4. After some preliminary and technical considerations we prove in Section 3 the main result of the paper, namely

Theorem 1.3 (Bitwisted Burnside-Frobenius theorem). Let $G$ be an Abelian group and $\phi$ and $\psi$ its endomorphisms. Then $R(\phi, \psi)=\# \operatorname{Coin}(\widehat{\phi}, \widehat{\psi})$ if one of these numbers is finite.

If $\psi$ is the identity map then the bitwisted Burnside-Frobenius theorem for Abelian groups implies the twisted Burnside-Frobenius theorem for Abelian groups from [13]: $R(\phi)=\# \operatorname{Fix}(\widehat{\phi})$ (the Reidemeister number of an endomorphism $\phi$ is equal to the number of fixed points of $\widehat{\phi}$ on the unitary dual, if one of these numbers is finite).

In some sense our theorem is a reply to a remark of J.-P. Serre [25], p. 34 that for compact infinite groups an analogue of the Burnside-Frobenius theorem is not interesting: $\infty=\infty$. It turns out that for infinite discrete groups the situation differs significantly, and even in non-twisted situations the number of classes can be finite (for one of the first examples see another book of J.-P. Serre [16]). A number of examples of groups and automorphisms with finite Reidemeister numbers were studied in [6], [11], [14], [18].

In Section 4.1 a bitwisted Dehn conjugacy problem is formulated and an affirmative answer to this problem for almost polycyclic groups is obtained.

Finally, in Section 5, based on [8], it is proved that for any two injective endomorphisms $\phi, \psi$ of a Baumslag-Solitar group $B(1, n)$ the Reidemeister number $R(\phi, \psi)$ is infinite. This is "opposite" case for the bitwisted Burnside-Frobenius theorem. It is an 
interesting open problem to describe all groups with the property that for any two injective endomorphisms $\phi, \psi$ the Reidemeister number $R(\phi, \psi)$ is infinite.

The interest in bitwisted and twisted conjugacy relations has its origins in the NielsenReidemeister coincidence and fixed point theory (see e.g. [6], [29]), in Selberg theory (see e.g. [1]), and Algebraic Geometry (see e.g. [19]).

The Lefschetz coincidence theorem generalizes the celebrated fixed point theorem to coincidences of two maps between closed connected orientable manifolds of the same dimension. H. Schirmer [24] developed a Nielsen-Reidemeister coincidence theory and proved a Wecken type theorem for coincidences. The analogous Lefschetz, Reidemeister and Nielsen coincidence numbers play a key role in the Nielsen-Reidemeister theory.

2. Compact groups. Let $G$ be a compact group and $\phi$ and $\psi$ its automorphisms. Hence $\widehat{G}$ is a discrete space. Then $C^{*}(G)=\oplus M_{i}$, where $M_{i}$ are the matrix algebras of irreducible representations. The infinite sum is in the following sense:

$$
C^{*}(G)=\left\{f_{i}\right\}, i \in\{1,2,3, \ldots\}=\hat{G}, f_{i} \in M_{i},\left\|f_{i}\right\| \rightarrow 0(i \rightarrow \infty) .
$$

When $G$ is finite and $\widehat{G}$ is finite this is exactly the Peter-Weyl theorem.

The characteristic function of a bitwisted class is a functional on $C^{*}(G)$. For a finite group it is evident, for a general compact group it is necessary to verify only the measurability of the bitwisted class with the respect to Haar measure, i.e. that bitwisted class is Borel. For a compact $G$, the bitwisted conjugacy classes being orbits of bitwisted action are compact and hence closed. Then its complement is open, hence Borel, and the class is Borel too.

Under the identification it passes to a sequence $\left\{\varphi_{i}\right\}$, where $\varphi_{i}$ is a functional on $M_{i}$ (the properties of convergence can be formulated, but they play no role at the moment). The conditions of invariance are the following: for each $\rho_{i} \in \widehat{G}$ one has $g\left[\varphi_{i}\right]=\varphi_{i}$, i.e. for any $a \in M_{i}$ and any $g \in G$ one has $\varphi_{i}\left(\rho_{i}(\psi(g)) a \rho_{i}\left(\phi\left(g^{-1}\right)\right)\right)=\varphi_{i}(a)$.

Let us recall the following well-known fact.

LEMMA 2.1. Each functional on the matrix algebra has the form $a \mapsto \operatorname{tr}(a b)$ for a fixed matrix $b$.

Proof. One has $\operatorname{dim}(M(n, \mathbb{C}))^{\prime}=\operatorname{dim}(M(n, \mathbb{C}))=n \times n$ and looking at matrices as at operators in $V, \operatorname{dim} V=n$, with base $e_{i}$, one can remark that the functionals $a \mapsto$ $\left\langle a e_{i}, e_{j}\right\rangle, i, j=1, \ldots, n$, are linearly independent. Hence, any functional takes the form

$$
a \mapsto \sum_{i, j} b_{i j}\left\langle a e_{i}, e_{j}\right\rangle=\sum_{i, j} b_{i j} a_{i}^{j}=\operatorname{tr}(b a), \quad b:=\left\|b_{i j}\right\| .
$$

THEOREM 2.2. Let $G$ be a compact group and $\phi$ and $\psi$ its automorphisms. Then $R(\phi, \psi)$ $=\# \operatorname{Coin}(\widehat{\phi}, \widehat{\psi})$ if one of these numbers is finite.

Proof. Let us consider invariant functionals on matrix algebras under bitwisted action of group $G$ :

$$
\begin{array}{ll}
\operatorname{tr}\left(b \rho_{i}(\psi(g)) a \rho_{i}\left(\phi\left(g^{-1}\right)\right)\right)=\operatorname{tr}(b a), & \forall a, g, \\
\operatorname{tr}\left(\left(b-\rho_{i}\left(\phi\left(g^{-1}\right)\right) b \rho_{i}(\psi(g))\right) a\right)=0, & \forall a, g,
\end{array}
$$


hence,

$$
b-\rho_{i}\left(\phi\left(g^{-1}\right)\right) b \rho_{i}(\psi(g))=0, \quad \forall g .
$$

Since $\rho_{i}$ is irreducible, the dimension of the space of such $b$ is 1 if $\rho_{i}$ is a coincidence point of $\widehat{\phi}$ and $\widehat{\psi}$ and 0 in the opposite case. So, we are done.

REMARK 2.3. In fact we are only interested in finite group case. Indeed, for a compact $G$, the bitwisted conjugacy classes being orbits of bitwisted action are compact and hence closed. If there is a finite number of them, then they are open too. Hence, the situation is more or less reduced to a discrete group: quotient by the component of unity.

REMARK 2.4. Bitwisted Burnside-Frobenius theorem for compact groups remains true if $\phi$ is an automorphism and $\psi$ is any endomorphism. It is equivalent to the twisted Burnside-Frobenius theorem for the one endomorphism $\phi^{-1} \circ \psi$ (see [15]).

EXAMPLE 2.5. The following counterexample to the bitwisted Burnside-Frobenius theorem for two endomorphisms of a nonabelian finite group was proposed to the author by E. Troitsky [27]. Let us consider two trivial endomorphisms $\phi$ and $\psi$ of a nonabelian finite group $G$, i.e. $\phi(g)=\psi(g)=e$ for every element $g \in G$. Then for every $x \in G$ and every $\gamma \in G$ we have $\psi(\gamma) x \phi\left(\gamma^{-1}\right)=x$, hence $R(\phi, \psi)=\# G$. On the other hand, $\widehat{\phi}(\rho)=\rho \circ \phi=I=\rho \circ \psi=\widehat{\psi}(\rho)$ for every $\rho \in \widehat{G}$ hence, if $G$ is nonabelian, then $\# \operatorname{Coin}(\widehat{\phi}, \widehat{\psi})=\# \widehat{G}$ and $R(\phi, \psi)=\# G \neq \# \widehat{G}=\# \operatorname{Coin}(\widehat{\phi}, \widehat{\psi})$.

3. Endomorphisms of Abelian groups. Let $\phi, \psi$ be endomorphisms of an Abelian group $G$.

Lemma 3.1. Let $G$ be Abelian. The bitwisted conjugacy class $H$ of $e$ is a subgroup. The other ones are cosets $g H$.

Proof. The first statement follows from the equalities

$$
\begin{gathered}
\left(\psi(h) \phi\left(h^{-1}\right) \psi(g) \phi\left(g^{-1}\right)=\psi(g h) \phi\left((g h)^{-1},\right.\right. \\
\left(\psi(h) \phi\left(h^{-1}\right)\right)^{-1}=\phi(h) \psi\left(h^{-1}\right)=\psi\left(h^{-1}\right) \phi(h) .
\end{gathered}
$$

For the second statement suppose $a \sim b$, i.e. $b=\psi(h) a \phi\left(h^{-1}\right)$. Then

$$
g b=g \psi(h) a \phi\left(h^{-1}\right)=\psi(h)(g a) \phi\left(h^{-1}\right), \quad g b \sim g a .
$$

Lemma 3.2. Suppose $u_{1}, u_{2} \in G$, and $\chi_{H}$ is the characteristic function of $H$ as a set. Then

$$
\chi_{H}\left(u_{1} u_{2}^{-1}\right)= \begin{cases}1, & \text { if } u_{1}, u_{2} \text { are in one coset }, \\ 0, & \text { otherwise. }\end{cases}
$$

Proof. Suppose $u_{1} \in g_{1} H, u_{2} \in g_{2} H$, hence, $u_{1}=g_{1} h_{1}, u_{2}=g_{2} h_{2}$. Then

$$
u_{1} u_{2}^{-1}=g_{1} h_{1} h_{2}^{-1} g_{2}^{-1} \in g_{1} g_{2}^{-1} H \text {. }
$$

Thus, $\chi_{H}\left(u_{1} u_{2}^{-1}\right)=1$ if and only if $g_{1} g_{2}^{-1} \in H$ and $u_{1}$ and $u_{2}$ are in the same class. Otherwise it is 0 .

The following lemma is well known.

LEMma 3.3. For any subgroup $H$ the function $\chi_{H}$ is of positive type. 
Proof. Let us take arbitrary elements $u_{1}, u_{2}, \ldots, u_{n}$ of $G$. Let us renumber them in such a way that some first are in $g_{1} H$, the next ones are in $g_{2} H$, and so on, till $g_{m} H$, where $g_{j} H$ are different cosets. By the previous lemma the matrix $\left\|p_{i t}\right\|:=\left\|\chi_{H}\left(u_{i} u_{t}^{-1}\right)\right\|$ is block-diagonal with square blocks formed by units. These blocks, and consequently the whole matrix are positive semi-definite.

LEMmA 3.4. In the Abelian case the characteristic functions of bitwisted conjugacy classes belong to the Fourier-Stieltjes algebra $B(G)=\left(C^{*}(G)\right)^{*}$.

Proof. By Lemma 3.1 in this case the characteristic functions of bitwisted conjugacy classes are the shifts of the characteristic function of the class $H$ of $e$. Hence, by Corollary (2.19) of [5], these characteristic functions are in $B(G)$.

Let us remark that there exists a natural isomorphism (Fourier transform)

$$
u \mapsto \widehat{u}, \quad C^{*}(G)=C_{r}^{*}(G) \cong C(\widehat{G}), \quad \widehat{g}(\rho):=\rho(g),
$$

(this is a number because irreducible representations of an Abelian group are 1-dimensional). In fact, it is better to look (for what follows) at the algebra $C(\widehat{G})$ as an algebra of continuous sections of a bundle of 1-dimensional matrix algebras over $\widehat{G}$.

Our characteristic functions, being in $B(G)=\left(C^{*}(G)\right)^{*}$ in this case, are mapped to the functionals on $C(\widehat{G})$ which, by the Riesz(-Markov-Kakutani) theorem, are measures on $\widehat{G}[\mathrm{C}]$. Which of these measures are invariant under the induced (bitwisted) action of $G$ ? Let us remark that an invariant non-trivial functional gives rise to at least one invariant space - its kernel.

Let us remark that convolution under the Fourier transform becomes point-wise multiplication. More precisely, the bitwisted action, for example, is defined as

$$
g[f](\rho)=\rho(\psi(g)) f(\rho) \rho\left(\phi\left(g^{-1}\right)\right), \quad \rho \in \widehat{G}, \quad g \in G, \quad f \in C(\widehat{G}) .
$$

There are 2 possibilities for the bitwisted action of $G$ on the representation algebra $\left.A_{\rho} \cong \mathbb{C}: 1\right)$ the linear span of the orbit of $1 \in A_{\rho}$ is equal to all $\left.A_{\rho}, 2\right)$ and the opposite case (the action is trivial).

The second case means that the space of intertwining operators between $A_{\widehat{\psi} \rho}$ and $A_{\widehat{\phi} \rho}$ equals $\mathbb{C}$, and $\rho$ is a coincidence point of $\widehat{\phi}$ and $\widehat{\psi}$. In the first case this is the opposite situation.

If we have a finite number of such coincidence points, then the space of bitwisted invariant measures is just the space of measures concentrated in these points. Indeed, let us describe the action of $G$ on measures in more detail.

Lemma 3.5. For any Borel set $E$ one has $g[\mu](E)=\int_{E} g[1] d \mu$.

Proof. The restriction of a measure to any Borel set commutes with the action of $G$, since the last is pointwise on $C(\widehat{G})$. For any Borel set $E$ one has

$$
g[\mu](E)=\int_{E} 1 d g[\mu]=\int_{E} g[1] d \mu .
$$

Hence, if $\mu$ is bitwisted invariant, then for any Borel set $E$ and any $g \in G$ one has

$$
\int_{E}(1-g[1]) d \mu=0
$$


Lemma 3.6. Suppose $f \in C(X)$, where $X$ is a compact Hausdorff space, and $\mu$ is a regular Borel measure on $X$, i.e. a functional on $C(X)$. Suppose, for any Borel set $E \subset X$ one has $\int_{E} f d \mu=0$. Then $\mu(h)=0$ for any $h \in C(X)$ such that $f(x)=0$ implies $h(x)=0$. I.e. $\mu$ is concentrated off the interior of supp $\mathrm{f}$.

Proof. Since the functions of the form $f h$ are dense in the space of functions $h$, it is sufficient to verify the statement for $f h$. Let us choose an arbitrary $\varepsilon>0$ and a simple function $h^{\prime}=\sum_{i=1}^{n} a_{i} \chi_{E_{i}}$ such that $\left|\mu\left(f h^{\prime}\right)-\mu(f h)\right|<\varepsilon$. Then

$$
\mu\left(f h^{\prime}\right)=\sum_{i=1}^{n} \int_{E_{i}} a_{i} f d \mu=\sum_{i=1}^{n} a_{i} \int_{E_{i}} f d \mu=0 .
$$

Since $\varepsilon$ is arbitrary, we are done.

Applying this lemma to a bitwisted invariant measure $\mu$ and $f=1-g[1]$ we obtain that $\mu$ is concentrated at our finite number of coincidence points of $\widehat{\phi}$ and $\widehat{\psi}$, because outside of them $f \neq 0$.

If we have an infinite number of coincidence points, then the space is infinite dimensional (we have an infinite number of measures concentrated in a finite number of points, each time different) and Reidemeister number is infinite as well. So, we have proved the following theorem:

THEOREM 3.7. Let $G$ be an Abelian group and $\phi$ and $\psi$ its endomorphisms. Then $R(\phi, \psi)$ $=\# \operatorname{Coin}(\widehat{\phi}, \widehat{\psi})$ if one of these numbers is finite.

\section{Automorphisms of bitwisted conjugacy separable groups, weak bitwisted} Burnside-Frobenius theorem. If the endomorphisms $\phi$ and $\psi$ are the automorphisms, then the bitwisted Burnside-Frobenius problem is equivalent to the twisted BurnsideFrobenius problem for the one automorphism $\phi^{-1} \circ \psi$. So we can apply all the results from [15], [16], [28] for one automorphism. In the present paper we define the following property for a countable discrete group $G$.

Definition 4.1. A group $G$ is called $(\phi, \psi)$-conjugacy separable with respect to endomorphisms $\phi, \psi: G \rightarrow G$ or bitwisted conjugacy separable, if any pair $g, h$ of non$(\phi, \psi)$-conjugate elements of $G$ are non- $(\bar{\phi}, \bar{\psi})$-conjugate in some finite quotient of $G$ respecting $\phi, \psi$.

If $\psi=i d$ and $\phi$ is an automorphism then this definition gives us the definition of $\phi$-conjugacy separable discrete group [15]. If both $\phi=i d$ and $\psi=i d$ then this definition gives us the well known notion of conjugacy separable group.

Now we formulate the main results for two automorphisms, namely

1. $(\phi, \psi)$-conjugacy separable groups and some extensions: Suppose, there is an extension $H \rightarrow G \rightarrow G / H$, respecting automorphisms $\phi$ and $\psi$ where the normal invariant subgroup $H$ is a $\left(\phi^{\prime}, \psi^{\prime}\right)$-conjugacy separable group; the Reidemeister number $R(\phi, \psi)$ is finite; $G / H$ is a finitely generated FC-group (i.e. a group with finite conjugacy classes). Then $G$ is a $(\phi, \psi)$-conjugacy separable group. 
2. Classes of $(\phi, \psi)$-conjugacy separable groups: almost polycyclic groups and finitely generated groups of polynomial growth are $(\phi, \psi)$-conjugacy separable groups if $\phi$ and $\psi$ are automorphisms.

3. Bitwisted Burnside-Frobenius theorem: Let $G$ be a $(\phi, \psi)$-conjugacy separable group and $\phi$ and $\psi$ its automorphisms. Denote by $\widehat{G}_{f}$ the subset of the unitary dual $\widehat{G}$ related to finite-dimensional representations and by $C_{f}(\phi, \psi)$ the number of coincidence points of $\widehat{\phi}_{f}$ and $\widehat{\psi}_{f}$ on $\widehat{G}_{f}$. Then $R(\phi, \psi)=C_{f}(\phi, \psi)$ if one of these numbers is finite.

4. The affirmative answer to the bitwisted Dehn conjugacy problem for polycyclic-byfinite groups (see subsection 4.1).

If $\psi$ is the identity map then the bitwisted Burnside-Frobenius theorem implies the twisted Burnside-Frobenius theorem from [15]: $R(\phi)=\# \operatorname{Fix}\left(\widehat{\phi}_{f}\right)$ (the Reidemeister number of an automorphism $\phi$ is equal to the number of finite-dimensional fixed points of $\widehat{\phi}$ on the unitary dual, if one of these numbers is finite).

In [13] one obtained from the twisted Burnside-Frobenius theorem the congruences for Reidemeister numbers which are similar to the remarkable Dold congruences for the Lefschetz numbers and which, together with the twisted Burnside-Frobenius theorem itself, are very important for the realization problem of Reidemeister numbers in topological dynamics and the study of the Reidemeister zeta-function.

We would like to emphasize the following important remarks.

1. In the original formulation by Fel'shtyn and Hill [10] the conjecture about twisted Burnside-Frobenius theorem asserts an equality of $R(\phi)$ and the number of fixed points of $\widehat{\phi}$ on $\widehat{G}$. This conjecture was proved in [10],[13] for f.g. type I groups.

2. As it follows from a key example, which we have studied in [14], a twisted conjugacy separable group can have infinite dimensional "supplementary" coincidence representations. More precisely we discuss in that paper the case of a semi-direct product of the action of $\mathbb{Z}$ on $\mathbb{Z} \oplus \mathbb{Z}$ by a hyperbolic automorphism with finite Reidemeister number (four to be precise) and the number of fixed points of $\widehat{\phi}$ on $\widehat{G}$ equal or greater than five, while the number of fixed points on $\widehat{G}_{f}$ is four.

This gives a counterexample to the conjecture in its original formulation and leads to the formulation using only fixed (or coincidence) points in $\widehat{G}_{f}$.

3. The origin of the phenomenon of an extra coincidence point lies in bad separation properties of $\widehat{G}$ for general discrete groups. A more deep study leads to the following general theorem.

Theorem 4.2 (Weak bitwisted Burnside-Frobenius theorem). Let $G$ be a countable discrete group and $\phi$ and $\psi$ its automorphisms. The number $R_{*}(\phi, \psi)$ of $(\phi, \psi)$-conjugacy classes related to bitwisted invariant functions on $G$ from the Fourier-Stieltjes algebra $B(G)$ is equal to the number $C_{*}(\phi, \psi)$ of generalized coincidence points of $\widehat{\phi}$ and $\widehat{\psi}$ on the Glimm spectrum of $G$, i.e. on the complete regularization of $\widehat{G}$, if one of the numbers $R_{*}(\phi, \psi)$ and $C_{*}(\phi, \psi)$ is finite.

4. Nevertheless for extreme groups (like Osin group [15], which is infinite finitely generated simple group with two conjugacy classes), even the modified conjecture is not 
true. Keeping in mind that for non-elementary Gromov hyperbolic groups, mapping class groups, saturated weakly branch groups and any two automorphisms $\phi, \psi$ the Reidemeister number $R(\phi, \psi)$ is infinite [7], [20], [9], [12] as well as for any two injective endomorphisms of Baumslag-Solitar groups cf. Section 5 and [8] while in the "opposite" case the bitwisted Burnside-Frobenius theorem is proved, we can hope that our results can lead to a complete resolution of the problem, if the extreme groups will be handled.

Let us remark that in some cases the weak bitwisted Burnside-Frobenius theorem easily implies the bitwisted Burnside-Frobenius theorem (in particular, in the form with finite-dimensional representations). For example, we will show directly that $R(\phi, \psi)=$ $R_{*}(\phi, \psi)$ in Abelian case. On the other hand, the unitary dual coincides with the Glimm spectrum.

4.1. Bitwisted Dehn conjugacy problem. The subject is closely related to some decision problem. Recall that M. Dehn in 1912 [4] has formulated in particular

Conjugacy problem. Does there exist an algorithm to determine whether an arbitrary pair of group words $U, V$ in the generators of $G$ define conjugate elements of $G$ ?

The following question was posed by G. Makanin [22] [Question 10.26(a)]:

Question. Does there exist an algorithm to determine whether for an arbitrary pair of group words $U$ and $V$ of a free group $G$ and an arbitrary automorphism $\phi$ of $G$ the equation $\phi(X) U=V X$ solvable in $G$ ?

In [3] an affirmative answer to Makanin's question is obtained.

In [2] the following problem, which generalizes the two above problems, was posed:

Twisted COnjugacy PROBlem. Does there exists an algorithm to determine whether for an arbitrary pair of group words $U$ and $V$ in the generators of $G$ the equality $\phi(X) U=V X$ holds for some $W \in G$ and $\phi \in H$, where $H$ is a fixed subset of $\operatorname{Aut}(G)$ ?

In [17] we proved that the twisted conjugacy problem has the affirmative answer for $G$ being a polycyclic-by-finite group and $H$ equal to a unique automorphism $\phi$.

In this article we suggest the following problem, which generalises the three above problems:

Bitwisted Conjugacy PRoblem. Does there exist an algorithm to determine whether for an arbitrary pair of group words $U$ and $V$ in the generators of $G$ the equality $\psi(X) U=$ $V \phi(X)$ holds for some $W \in G$ and endomorphisms $\phi, \psi \in H$, where $H$ is a fixed subset of $\operatorname{End}(G)$ ?

THEOREM 4.3. The bitwisted conjugacy problem has the affirmative answer for $G$ being an almost polycyclic group and $H$ equal to a pair of automorphisms $\phi, \psi$.

Proof. This follows immediately from $(\phi, \psi)$-conjugacy separability of almost polycyclic groups by the same argument as in the paper of Mal'cev [21] (see also [23], where the property of conjugacy separability was first formulated) for the (non-twisted) conjugacy problem. 
5. Baumslag-Solitar groups $B(1, n)$. In this section based on (a part of) [8] it is proved that for any two injective endomorphisms $\phi, \psi$ of a Baumslag-Solitar group $B(1, n)$ the Reidemeister number $R(\phi, \psi)$ is infinite. From [8] the similar result for two injective endomorphisms of $B(m, n)$ where $|n| \neq|m|$ and $|n m| \neq 0,1$ follows as well. For this class of groups there is no bitwisted Burnside-Frobenius theorem (the Reidemeister number $R(\phi, \psi)$ is infinite). It is an open problem to describe all groups with this property.

The presentation in this section is selfcontained.

Let $B(1, n)=\left\langle a, b: a^{-1} b a=b^{n}, n>1\right\rangle$ be the Baumslag-Solitar groups. These groups are finitely-generated solvable groups (in particular amenable) which are not virtually nilpotent. These groups have exponential growth and they are not Gromov hyperbolic. Furthermore, these groups are torsion free and metabelian (an extension of an Abelian group by an Abelian one). More precisely one has

Proposition 5.1. $B(1, n) \cong \mathbb{Z}[1 / n] \rtimes_{\theta} \mathbb{Z}$, where the action of $\mathbb{Z}$ on $\mathbb{Z}[1 / n]$ is given by $\theta(1)(x)=x / n$.

Proof. The map defined by $\iota(a)=(0,1)$ and $\iota(b)=(1,0)$ extends to a unique homomorphism $\iota: B(1, n) \rightarrow \mathbb{Z}[1 / n] \rtimes \mathbb{Z}$, because

$$
\iota\left(a^{-1}\right) * \iota(b) * \iota(a)=(0,-1) *(1,0) *(0,1)=(0,-1) *(1,1)=(n, 0)=\iota\left(b^{n}\right) .
$$

One has

$$
\iota\left(a^{r} b^{s} a^{-r}\right)=(0, r) *(s,-r)=\left(\frac{s}{n^{r}}, 0\right) .
$$

The map $\iota$ is clearly surjective. Let us show that this homomorphism is injective. Let us remark that the group relation implies $a^{-1} b^{-1} a=b^{-n}$. Hence for any $s$ one has $a^{-1} b^{s} a=b^{n s}$. Thus we can move all $a^{-1}$ to the right (until they annihilate with some $a$ or take the extreme right place) and all $a$ to the left, with an appropriate changing of powers of $b$. Hence any word in $B(1, n)$ is equivalent to a word of the form $w=a^{r_{1}} b^{s} a^{r_{2}}$, where $r_{1} \geq 0, r_{2} \leq 0$. Then $\iota(w)=\left(m, r_{1}+r_{2}\right)$ for some $m \in \mathbb{Z}[1 / n]$. Hence, if $r_{1}+r_{2} \neq 0$, then $\iota(w) \neq e$. Let now $r_{1}+r_{2}=0$, then by $(1)$ if $\iota(w)=e$, then $s=0$ and $w=e$.

Consider the homomorphism ||$_{a}: B(1, n) \rightarrow \mathbb{Z}$ which associates to each word $w \in B(1, n)$ the sum of the exponents of $a$ in this word. Since this sum for the relation is zero, this is a well defined map, which is evidently surjective.

Proposition $5.2([8])$. We have a short exact sequence

$$
0 \rightarrow K \rightarrow B(1, n) \stackrel{\mid}{\rightarrow} \rightarrow \mathbb{Z} \rightarrow 1,
$$

where $K$ is the kernel of ||$_{a}$. Moreover, $B(1, n)$ equals a semidirect product $K \rtimes \mathbb{Z}$.

Proof. The first statement follows from surjectivity of $|\cdot|_{a}$. Since $\mathbb{Z}$ is free, this sequence splits.

Proposition 5.3 ([8]). The kernel $K$ coincide with the normalizer $N\langle b\rangle$ of the subgroup $\langle b\rangle$ generated by $b$ in $B(1, n)$ :

$$
0 \rightarrow N\langle b\rangle \rightarrow B(1, n)^{\mid} \stackrel{\mid}{\rightarrow} \mathbb{Z} \rightarrow 1 .
$$

Proof. We have $N\langle b\rangle \subset K$. The quotient $B(1, n) / N\langle b\rangle$ has the following presentation: $\bar{a}^{-1} \bar{b} \bar{a}=\bar{b}^{n}, \bar{b}=1$. Therefore this group is isomorphic to $\mathbb{Z}$ under the identification 
$[a] \leftrightarrow 1_{\mathbb{Z}}$. Hence the natural projection coincides with the map ||$a$ and we have the following commutative diagram

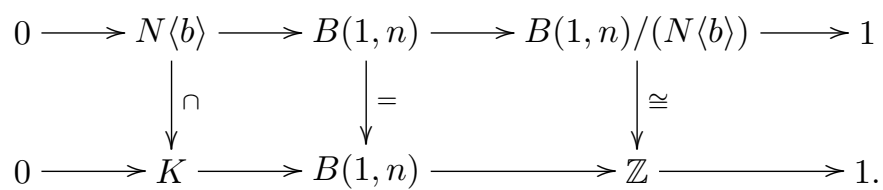

The five-lemma completes the proof.

Proposition 5.4 ([8]). Any endomorphism $\phi: B(1, n) \rightarrow B(1, n)$ is an endomorphism of the short exact sequence (2).

Proof. Let $\bar{\phi}$ be the endomorphism induced by $\phi$ on the abelianization $B(1, n)_{a b}$ of $B(1, n)$. The group $B(1, n)_{a b}$ is isomorphic to $Z_{n-1}+Z$. The torsion elements of $B(1, n)_{a b}$ form a subgroup isomorphic to $Z_{n-1}$ which is invariant under any endomorphism. The preimage of this subgroup under the projection $B(1, n) \rightarrow B(1, n)_{a b}$ is exactly the subgroup $N(b)$, i.e. the elements represented by words where the sum of the powers of $a$ is zero. So it follows that $N(b)$ is mapped into $N(b)$ and the result follows.

TheOREm 5.5 ([8]). For any two injective endomorphisms $\phi$ and $\psi$ of $B(1, n)$ the Reidemeister number $R(\phi, \psi)$ is infinite.

Proof. By Proposition 5.4 they are endomorphisms of the short exact sequence. The induced maps $\bar{\phi}$ and $\bar{\psi}$ on the quotient are injective endomorphisms of $\mathbb{Z}$. If $\bar{\phi}=\operatorname{Id}_{\mathbb{Z}}$, and $\bar{\psi}=\operatorname{Id}_{\mathbb{Z}}$, then by [8] the number of bitwisted classes is infinite. Hence,at least one of $\bar{\phi}$ or $\bar{\psi}$ is multiplication by $k \neq 0,1$. But this is impossible. Indeed, when we apply $\phi$ to the relation $a^{-1} b a=b^{n}$, under the identification of Proposition $5.1 \iota: B(1, n) \cong \mathbb{Z}[1 / n] \rtimes \mathbb{Z}$ we have, because $\phi(b) \in N\langle b\rangle$ and hence $\iota(\phi(b))=(d, 0)$ for some $d \in \mathbb{Z}[1 / n]$,

$$
(n d, 0)=\iota\left(\phi\left(b^{n}\right)\right)=\iota\left(\phi\left(a^{-1} b a\right)\right)=\iota\left(a^{-k} \phi(b) a^{k}\right)=\left(d \cdot n^{k}, 0\right) .
$$

This implies that either $n^{1-k}=1$ or $\phi(b)=0$.

Acknowledgements. I would like to thank the MPI for its kind support and hospitality while the most part of this work has been completed.

The author is grateful to D. Gonçalves, R. Hill, E. Troitsky, L. Vainerman and A. Vershik for helpful discussions.

The author is also indebted to the referee for a careful reading and valuable suggestions.

\section{References}

[1] J. Arthur and L. Clozel, Simple Algebras, Base Change, and the Advanced Theory of the Trace Formula, Princeton University Press, Princeton, NJ, 1989.

[2] V. Bardakov, L. Bokut, and A. Vesnin, Twisted conjugacy in free groups and Makanin's question, Southeast Asian Bull. Math. 29 (2005), 209-226.

[3] O. Bogopolski, A. Martino, O. Maslakova, E. Ventura, Free-by-cyclic groups have solvable conjugacy problem, Bulletin of the London Mathematical Society 38 (2006), 787-794.

[4] A. Connes, Noncommutative Geometry, Academic Press, 1994. 
[5] M. Dehn, Über unendliche diskontinuierliche Gruppen, Math. Ann. 71 (1911), 116-144.

[6] P. Eymard, L'algèbre de Fourier d'un groupe localement compact, Bull. Soc. Math. France 92 (1964), 181-236.

[7] A. Fel'shtyn, Dynamical zeta functions, Nielsen theory and Reidemeister torsion, Mem. Amer. Math. Soc. 147 (2000), no. 699.

[8] A. L. Fel'shtyn, The Reidemeister number of any automorphism of a Gromov hyperbolic group is infinite, Zap. Nauchn. Sem. S.-Peterburg. Otdel. Mat. Inst. Steklov. (POMI) 279 (2001), no. 6 (Geom. i Topol.), 229-240, 250.

[9] A. Fel'shtyn and D. Gonçalves, The Reidemeister number of any automorphism of a Baumslag-Solitar group is infinite, in: Geometry and Dynamics of Groups and Spaces, Progress in Mathematics 265, Birkhäuser, 2008, 399-414.

[10] A. Fel'shtyn and D. L. Gonçalves, Twisted conjugacy classes in symplectic groups, mapping class groups and braid groups (with Appendix written with F. Dahmani), arxiv: math.GR/0708.2628.

[11] A. Fel'shtyn and R. Hill, The Reidemeister zeta function with applications to Nielsen theory and a connection with Reidemeister torsion, K-Theory 8 (1994), 367-393.

[12] A. Fel'shtyn, R. Hill, and P. Wong, Reidemeister numbers of equivariant maps, Topology Appl. 67 (1995), no. 2, 119-131.

[13] A. Fel'shtyn, Y. Leonov and E. Troitsky, Reidemeister numbers of saturated weakly branch groups, Geometria Dedicata 134(2008), 61-73.

[14] A. Fel'shtyn and E. Troitsky, A twisted Burnside theorem for countable groups and Reidemeister numbers, in: Noncommutative Geometry and Number Theory (Bonn, 2003), K. Consani and M. Marcolli (eds.), Vieweg, Braunschweig, 2006, 141-154.

[15] A. Fel'shtyn, E. Troitsky, and A. Vershik, Twisted Burnside theorem for type II 1 groups: an example, Mathematical Research Letters 13 (2006), 719-728.

[16] A. Fel'shtyn and E. Troitsky, Twisted Burnside-Frobenius theory for discrete groups, J. Reine Angew. Math. 613 (2007), 193-210.

[17] A. Fel'shtyn and E. Troitsky, Geometry of Reidemeister classes and twisted Burnside theorem, Journal of K-theory 2 (2008), 405-445.

[18] A. Fel'shtyn and E. Troitsky, Twisted conjugacy separable groups, arXiv:math. GR/0606764, 2006.

[19] D. Gonçalves and P. Wong, Twisted conjugacy classes in exponential growth groups, Bull. London Math. Soc. 35 (2003), 261-268.

[20] A. Grothendieck, Formules de Nielsen-Wecken et de Lefschetz en géométrie algébrique, in: Séminaire de Géométrie Algébrique du Bois-Marie 1965-66. SGA 5, Lecture Notes in Math. 569, Springer-Verlag, Berlin, 1977, 407-441.

[21] G. Levitt and M. Lustig, Most automorphisms of a hyperbolic group have very simple dynamics, Ann. Scient. Éc. Norm. Sup. 33 (2000), 507-517.

[22] A. I. Malcev, On homomorphisms onto finite groups, Uchen. Zapiski Ivanovsk. Ped. Instituta 18 (1958), no. 5, 49-60 (= Selected Papers, Vol. 1, 1976, 450-461).

[23] V. D. Mazurov and E. I. Khukhro (eds.), The Kourovka notebook, augmented ed., Ros. Akademiya Nauk, Sibirskoe Otdelenie, Institut Matematiki im. S. L. Soboleva, Novosibirsk, 2002, Unsolved problems in group theory.

[24] A. W. Mostowski, On the decidability of some problems in special classes of groups, Fund. Math. 59 (1966), 123-135.

[25] H. Schirmer, Mindestzahlen von Koinzidenzpunkten. J. Reine Angew. Math. 194 (1955), 21-39. 
[26] J.-P. Serre, Linear Representations of Finite Groups, Springer, New York, 1977.

[27] J.-P. Serre, Trees, Springer, Berlin, 2003.

[28] E. Troitsky, private communication.

[29] E. Troitsky, Noncommutative Riesz-Markov-Kakutani theorem and weak twisted Burnside theorem, Funct. Anal. Appl., to appear.

[30] P. Wong, Reidemeister number, Hirsch rank, coincidences on polycyclic groups and solvmanifolds, J. Reine Angew. Math. 524 (2000), 185-204. 